\title{
The topological entropy versus level sets for interval maps (part II)
}

\author{
by \\ Jozef Bobok (Praha)
}

\begin{abstract}
Let $f:[a, b] \rightarrow[a, b]$ be a continuous function of the compact real interval such that (i) card $f^{-1}(y) \geq 2$ for every $y \in[a, b]$; (ii) for some $m \in\{\infty, 2,3, \ldots\}$ there is a countable set $L \subset[a, b]$ such that card $f^{-1}(y) \geq m$ for every $y \in[a, b] \backslash L$. We show that the topological entropy of $f$ is greater than or equal to $\log m$. This generalizes our previous result for $m=2$.
\end{abstract}

0. Introduction. The aim of this paper is to demonstrate a relationship of two characteristics of an interval map: its topological entropy and cardinalities of level sets. Our main result states that for an interval map - as opposed to circle maps or some maps on higher dimensional manifolds [Ma] - the cardinalities of level sets strongly determine the value of entropy. Elaborating our approach from [B1] we focus on the case of $m$-preimages for fixed $m \in \mathbb{N} \backslash\{1\}$ or $m$ equal to infinity. In particular, Theorem 4.3 shows that if we forbid an exceptional case of one-point level sets, the dependence between entropy and the cardinalities of level sets is rather regular. Based on that and several known (always) non-transitive counterexamples we conjecture that this should be the case for a wider variety of transitive maps on compact topological manifolds.

Let $[a, b]$ be a compact real interval. We denote by $C([a, b])$ the set of all continuous functions which map $[a, b]$ into itself. Any element of $C([a, b])$ is called an interval map. For $m \in\{\infty, 2,3,4, \ldots\}$ let $L(m,[a, b])$ be the subset of $C([a, b])$ maps satisfying

$$
\forall y \in[a, b]: \quad \operatorname{card} f^{-1}(y) \geq m .
$$

From [B1] we know that the topological entropy of any $f \in L(2,[a, b])$ is greater than or equal to $\log 2$. In this paper we extend that result as follows.

2000 Mathematics Subject Classification: 37E05, 37B40.

Key words and phrases: interval map, level set, topological entropy.

Research supported by Grant Agency of the Czech Republic, contract n. 201/00/0859. 
Let $L^{\sharp}(m,[a, b])$ be the subset of $L(2,[a, b])$ defined by $\left(1_{m}^{\sharp}\right) \quad \forall f \in L^{\sharp}(m,[a, b]) \exists L \subset[a, b], L$ countable $\forall y \in[a, b] \backslash L:$ card $f^{-1}(y) \geq m$.

We show the following statement.

TheOREM 4.3. Let $f \in L^{\sharp}(m,[a, b])$. Then the topological entropy of $f$ is greater than or equal to $\log m$. In particular, this is true for any map from $L(m,[a, b]) \subset L^{\sharp}(m,[a, b])$.

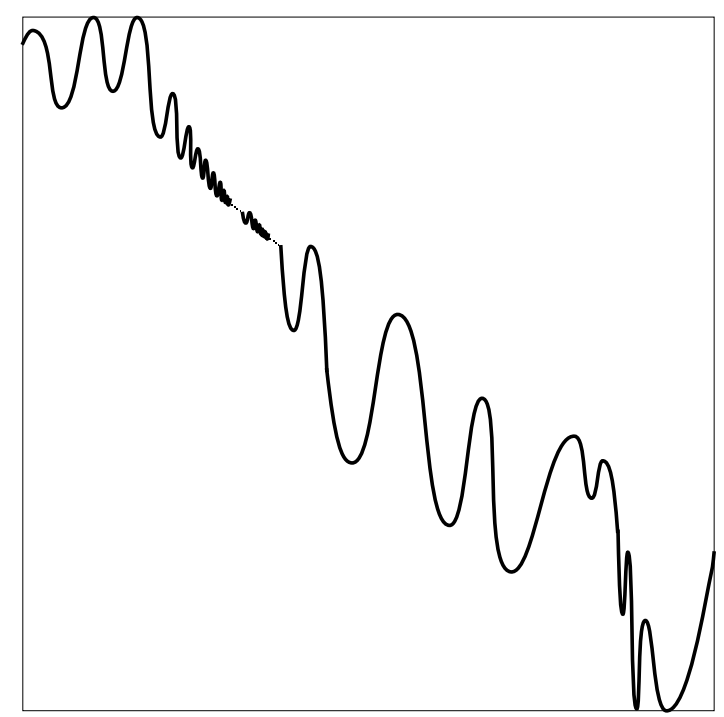

Fig. 1. $f \in L^{\sharp}(3,[a, b]), \operatorname{ent}(f) \geq \log 3$

Our main result is rather delicate. One can easily find an interval map of entropy zero that does not satisfy $\left(1_{\infty}\right)$ for exactly one point from $[a, b]$.

In this paper we use several times the following type of "horseshoe".

Definition 0.1. Let $(X, \varrho)$ be a compact metric space, $f: X \rightarrow X$ be continuous and $S_{0}, S_{1}, \ldots, S_{m-1} \subset X$ be closed. We say that the sets $S_{0}, S_{1}, \ldots, S_{m-1}$ form an $m$-horseshoe if they are pairwise disjoint and

$$
f\left(S_{0}\right) \cap f\left(S_{1}\right) \cap \cdots \cap f\left(S_{m-1}\right) \supset S_{0} \cup S_{1} \cup \cdots \cup S_{m-1} .
$$

As an easy consequence of the definition of topological entropy we obtain the following [DGS].

Proposition 0.2. Let $(X, \varrho)$ be a compact metric space and $f: X \rightarrow X$ be continuous. If the sets $S_{0}, S_{1}, \ldots, S_{m-1} \subset X$ form an m-horseshoe then the topological entropy of $f$ is greater than or equal to $\log m$. 


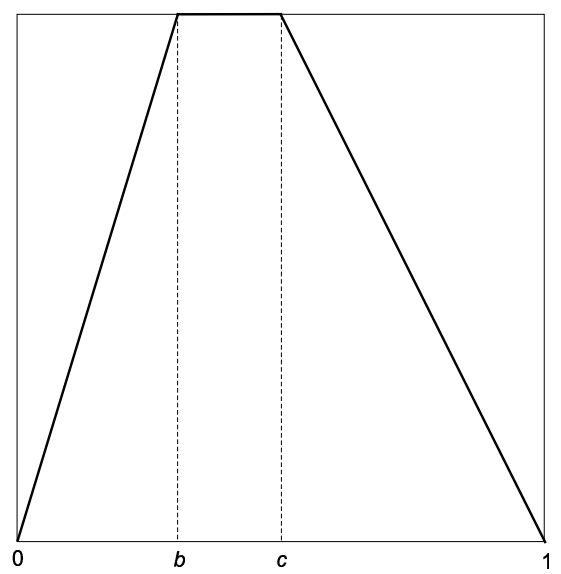

Fig 2. The sets $S_{0}=[0, b], S_{1}=[c, 1]$ form a 2-horseshoe

The paper is organized as follows. In Section 1 we give some basic notation, definitions and known results (Theorems 1.1 and 1.3). Section 2 is devoted to the lemmas used throughout the paper. In Section 3 we analyze the properties of maps from $L^{\sharp}(m,[a, b]), m \in\{\infty, 2, \ldots\}$. In Section 4 we prove the key Lemma 4.1, Corollary 4.2 and Theorem 4.3.

Finally, Section 5 is devoted to an application of Theorem 4.3. We show that the entropy of a Besicovitch function (preserving the Lebesgue measure) is infinite.

1. Definitions and known results. By $\mathbb{N}$ we denote the set of positive integers. We will work with topological dynamics $(X, T)$, where $X$ is a compact metric space and $T: X \rightarrow X$ is a continuous map. $(X, T)$ is minimal if $\left\{T^{i}(x): i \in \mathbb{N}\right\}$ is dense in $X$ for each $x \in X$. A subset $M$ of $X$ is $T$-invariant if $T(M) \subset M$, and minimal (in $X$ ) if $M$ is closed, $T$-invariant and $(M, T \mid M)$ is minimal.

Let $\varrho$ be a metric on the space $X$. We will use Bowen's definition of topological entropy [DGS]. A set $E \subset X$ is $(n, \varepsilon)$-separated (with respect to $T)$ if, whenever $x, y \in E, x \neq y$ then $\max _{0 \leq i \leq n-1} \varrho\left(T^{i}(x), T^{i}(y)\right)>\varepsilon$. For a compact set $K \subset X$ we denote by $s(n, \varepsilon, K)$ the largest cardinality of any $(n, \varepsilon)$-separated subset of $K$. Put

$$
\operatorname{ent}(T, K)=\lim _{\varepsilon \rightarrow 0^{+}} \limsup _{n \rightarrow \infty} \frac{1}{n} \log s(n, \varepsilon, K)
$$

and $\operatorname{ent}(T)=\operatorname{ent}(T, X)$. The quantity $\operatorname{ent}(T)$ is called the topological entropy of $T$.

A topological dynamics $(Y, S)$ is a factor of $(X, T)$ if there is a continuous surjective factor map $h: X \rightarrow Y$ such that $h \circ T=S \circ h$. 
TheOrem $1.1([\mathrm{Bo}])$. If $(Y, S)$ is a factor of $(X, T)$ then

$$
\operatorname{ent}(S) \leq \operatorname{ent}(T) \leq \operatorname{ent}(S)+\sup _{y \in Y} \operatorname{ent}\left(T, h^{-1}(\{y\})\right) .
$$

As usual, the $\omega$-limit set $\omega_{T}(x)$ of $x \in X$ consists of all the limit points of $\left\{T^{i}(x): i \in \mathbb{N}\right\}$. A set $P \subset X$ is called a periodic orbit (of period $n$ ) if $P=\left\{x, T(x), \ldots, T^{n-1}(x)\right\}$ for some $x \in X$ and $n \in \mathbb{N}$ with $T^{n}(x)=x$. A normalized Borel measure $\mu$ on $X$ is $T$-invariant if $\mu\left(T^{-1}(E)\right)=\mu(E)$ for each Borel set $E \subset X$.

Now we list several useful properties of minimal sets. As is well known they can be considered in any topological dynamics.

Lemma 1.2. (i) For each $x \in X$, the $\omega$-limit set $\omega_{T}(x)$ contains some minimal set.

(ii) Any minimal set in $X$ is either finite and then a periodic orbit of $T$, or infinite and then uncountable.

(iii) If $(X, T)$ is minimal and a measure $\mu$ on $X$ is $T$-invariant then either $X$ is finite and then $\mu$ is atomic, or $X$ is infinite and then $\mu$ is nonatomic. In any case $\operatorname{supp} \mu=X$.

(iv) Let $M \subset X$ be minimal in $X$. If $M$ is infinite then for each countable closed set $C \subset M$ and $x \in M$ we have $\lim _{n} n^{-1} \operatorname{card}\{0 \leq i \leq$ $\left.n-1: T^{i}(x) \in C\right\}=0$.

Proof. See $[\mathrm{BC}]$ for (i)-(iii).

Let us prove (iv). Notice that by our assumption and (ii), $M$ is uncountable. Suppose to the contrary there is an increasing sequence $\left\{k_{n}\right\}_{n=1}^{\infty}$ such that $\lim _{n} k_{n}^{-1} C\left(k_{n}, x\right)=a \in(0,1]$, where $C(n, x)=\operatorname{card}\{0 \leq i \leq$ $n-1: T^{i}(x) \in C$ \}. Then using the standard method [DGS, Prop. 2.7] we can find an atomic $T$-invariant measure $\mu$ for which $\mu(C)>0$ and $\operatorname{supp} \mu \subsetneq M$, a contradiction with (ii) and (iii).

We will use the symbolic dynamics [DGS]. For $m \in \mathbb{N}$ consider the set $N_{m}=\{0,1, \ldots, m-1\}$ as a space with the discrete topology, and denote by $\Omega_{m}$ the infinite product space $\prod_{i=0}^{\infty} X_{i}$, where $X_{i}=N_{m}$ for all $i$. The shift map $\sigma_{m}: \Omega_{m} \rightarrow \Omega_{m}$ (in what follows we write $\sigma$ instead of $\sigma_{m}$ ) is defined by $(\sigma(\omega))_{i}=\omega_{i+1}$ for $i \in \mathbb{N} \cup\{0\}$. Obviously, each $\left(\Omega_{m}, \sigma\right)$ is a topological dynamics.

It is known [DGS, Prop. 16.11] that for $\Omega \subset \Omega_{m}$ closed,

$$
\operatorname{ent}(\sigma, \Omega)=\lim _{n} \frac{1}{n} \log \operatorname{card} \Omega(n),
$$

where $\Omega(n)=\left\{\omega(n)=\left(\omega_{0}, \ldots, \omega_{n-1}\right): \omega \in \Omega\right\}$.

The following remarkable result concerns the topological entropy of subshifts in $\left(\Omega_{m}, \sigma\right)$. 
TheORem $1.3([\mathrm{G}])$. Let $m \in \mathbb{N}$. For any positive $\varepsilon$ there is a minimal set $\Gamma$ in $\Omega_{m}$ such that $\operatorname{ent}(\sigma, \Gamma)>-\varepsilon+\log m$.

The following easy lemma is needed in the proof of Theorem 4.3. Put $\Omega_{j, k}=\left\{\omega \in \Omega_{m}: \omega_{2 i+j} \neq k\right.$ for each $\left.i \in \mathbb{N} \cup\{0\}\right\}$ for $j \in N_{2}, k \in N_{m}$, and

$$
\Omega(M(\infty))=\bigcup_{(j, k) \in N_{2} \times N_{m}} \Omega_{j, k} .
$$

Lemma 1.4. Let $\Omega=\Omega(M(\infty))$ be as in (3).

(i) The set $\Omega$ is closed $\sigma$-invariant in $\Omega_{m}$ and $\operatorname{ent}(\sigma, \Omega)=\frac{1}{2} \log [m(m-1)]$.

(ii) For each $k_{0} \geq 2$,

$$
\frac{1}{2} \log [m(m-1)] \leq \frac{1}{k_{0}} \log (m-1)+\frac{k_{0}-1}{k_{0}} \log m .
$$

Proof. (i) The closedness of $\Omega$ is clear. Since $\sigma(\Omega) \subset \Omega$, we can compute the entropy $\operatorname{ent}(\sigma, \Omega)$ using (2). Obviously, for each $n \in \mathbb{N}$ and $(j, k) \in$ $N_{2} \times N_{m}$ we have card $\Omega_{j, k}(2 n)=[m(m-1)]^{n}$, hence the conclusion follows. Property (ii) is clear.

2. Lemmas. In what follows, by $[a, b]$, resp. $(a, b)$ we always denote a compact, resp. open real interval. As usual, a map $f \in C([a, b])$ has a strict local maximum, resp. minimum at a point $x \in[a, b]$ if there is an $\varepsilon>0$ such that for each $y \in[a, b] \cap((x-\varepsilon, x+\varepsilon) \backslash\{x\})$ we have $f(y)<f(x)$, resp. $f(y)>f(x)$. In this case we say that $f(x)$ is a locally extremal value. We set $C_{\text {extrem }}(f)=\{y \in[a, b]: y$ is a locally extremal value $\}$ and $C_{\text {inter }}(f)=$ $\left\{y \in[a, b]: f^{-1}(y)\right.$ contains an interval $\}$.

The following lemma is well known.

Lemma 2.1. Let $f \in C([a, b])$. Then the set $C_{\text {inter }}(f) \cup C_{\text {extrem }}(f)$ is countable.

Proof. Obviously $C_{\text {inter }}(f)$ is countable. Moreover, there is a countable set of points in $[a, b]$ where a map $f$ attains its strict local extreme $[\mathrm{Br}]$, hence also $C_{\text {extrem }}(f)$ is countable.

Let $\mathcal{J}=\left\{J_{\alpha}\right\}_{\alpha}$ and $\mathcal{K}=\left\{K_{\beta}\right\}_{\beta}$ be two systems of open subintervals of $(a, b)$. Then $\mathcal{K}$ is said to be finer than $\mathcal{J}$ if every $K_{\beta}$ is contained in some $J_{\alpha}$. In what follows by a countable set we also mean a finite one.

Lemma 2.2. (i) For any $T \subset \mathbb{R}$ the set $\{x \in T: x$ is a one-sided limit point of $T\}$ is countable.

(ii) Let $\mathcal{J}=\left\{J_{\alpha}\right\}_{\alpha}$ be a system of open subintervals of $(a, b)$ for which $(a, b) \backslash \bigcup_{\alpha} J_{\alpha}$ is countable. There is a countable system $\mathcal{K}=\left\{K_{n}\right\}_{n}$ of pairwise disjoint open subintervals of $(a, b)$ that is finer than $\mathcal{J}$ and such that $(a, b) \backslash \bigcup_{n} K_{n}$ is countable. 
Proof. Conclusion (i) is clear.

Let us prove (ii). Since each $J_{\alpha}$ can be expressed as an increasing union of open intervals with rational endpoints, there is a countable system $\mathcal{L}=$ $\left\{L_{n}\right\}_{n \in \mathbb{N}}$ of open intervals which is finer than $\mathcal{J}$ and such that $(a, b) \backslash$ $\bigcup_{n \in \mathbb{N}} L_{n}=(a, b) \backslash \bigcup_{\alpha} J_{\alpha}$. To construct $\mathcal{K}$, in the first step we put $K_{1}=L_{1}$. Suppose we have already defined open intervals $K_{1}, \ldots, K_{l}$ in $m-1$ steps; then the new open intervals from $\mathcal{K}$ given by the $m$ th step are the nonempty connected components of $L_{m} \backslash \overline{\bigcup_{i=1}^{l} K_{i}}$. Now the reader can verify that the resulting countable system $\mathcal{K}$ satisfies (ii).

As usual, for $y \in[a, b]$ by a left neighbourhood of $y$ in the relative topology we mean any set containing an interval $(y-\delta, y] \cap[a, b]$ with some $\delta$ positive; right and two-sided neighbourhoods are defined analogously.

Definition. Let $f \in C([a, b])$. We say that $x \in[a, b]$ is left regular if for each two-sided neighbourhood $U(x)$ of $x$ the set $f(U(x))$ is a left neighbourhood of $f(x)$; a right regular, resp. regular point is defined analogously. We denote the corresponding sets of regular points by $R_{\text {lreg }}(f), R_{\text {rreg }}(f), R_{\text {reg }}(f)$ respectively. Obviously, $R_{\text {reg }}(f)=R_{\text {Ireg }}(f) \cap R_{\text {rreg }}(f)$.

For $f \in C([a, b])$ we define the following sets (see Lemma 2.1):

$$
\begin{aligned}
C_{\text {reg }}(f)=\{y \in(a, b): & \left.\operatorname{card}\left(f^{-1}(y) \cap R_{\text {reg }}(f)\right) \geq m\right\}, \\
C_{\text {rreg }}(f)=\{y \in(a, b): & y \notin C_{\text {reg }} \cup C_{\text {extrem }} \cup C_{\text {inter }} \\
& \left.\& \operatorname{card}\left(f^{-1}(y) \cap R_{\text {rreg }}(f)\right) \geq m\right\}, \\
C_{\text {lreg }}(f)=\{y \in(a, b): & y \notin C_{\text {rreg }} \cup C_{\text {reg }} \cup C_{\text {extrem }} \cup C_{\text {inter }} \\
& \left.\& \operatorname{card}\left(f^{-1}(y) \cap R_{\text {lreg }}(f)\right) \geq m\right\} .
\end{aligned}
$$

For $y \in[a, b]$ we put $T(y)=\left\{\left(t_{0}, \ldots, t_{m-1}\right): t_{0}<\cdots<t_{m-1} \& t_{i} \in\right.$ $\left.f^{-1}(y)\right\} \subset[a, b]^{m}$ and fix a map $\phi: C_{\text {lreg }}(f) \cup C_{\text {rreg }}(f) \cup C_{\text {reg }}(f) \rightarrow[a, b]^{m}$ satisfying

$$
\begin{array}{r}
\phi(y)=\left(t_{0}, \ldots, t_{m-1}\right) \in T(y), \quad t_{i} \in f^{-1}(y) \cap R_{j}(f) \text { if } y \in C_{j}(f), \\
j \in\{\text { lreg, rreg, reg }\} .
\end{array}
$$

The next lemma will be important when proving our main result. We use the notation $C=C_{\text {lreg }}(f) \cup C_{\text {rreg }}(f) \cup C_{\text {reg }}(f), N_{m}=\{0,1, \ldots, m-1\}$; for $t \in \mathbb{R}^{m}$ we put $\|t\|=\min _{0 \leq i \leq m-2}\left|t_{i+1}-t_{i}\right|$, and for a map $f, y \in \mathbb{R}$ and $\varepsilon>0$,

$$
J(\varepsilon, y)= \begin{cases}(y-\varepsilon, y), & y \in C_{\mathrm{lreg}}(f), \\ (y, y+\varepsilon), & y \in C_{\mathrm{rreg}}(f), \\ (y-\varepsilon, y+\varepsilon), & y \in C_{\mathrm{reg}}(f) .\end{cases}
$$


Lemma 2.3. Let $f \in C([a, b])$.

(i) If $y \in[a, b]$ and $y \notin C_{\text {inter }}(f)$ then $f^{-1}(y) \subset R_{\operatorname{lreg}}(f) \cup R_{\operatorname{rreg}}(f)$.

(ii) For any $y^{\prime} \in C$ there is an $\varepsilon\left(y^{\prime}\right)>0$ such that $J\left(\varepsilon\left(y^{\prime}\right), y^{\prime}\right) \subset(a, b)$ and

$\forall y \in J\left(\varepsilon\left(y^{\prime}\right), y^{\prime}\right) \exists t \in T(y) \forall i \in N_{m}: \quad t_{i} \in\left(\phi\left(y^{\prime}\right)_{i}-\delta, \phi\left(y^{\prime}\right)_{i}+\delta\right)$, where $\delta=\left\|\phi\left(y^{\prime}\right)\right\| / 100($ see $(\star))$.

In statements (iii)-(iv) we assume that the set $(a, b) \backslash C$ is countable.

(iii) There exists a countable system $\left\{K_{n}\right\}_{n}$ of pairwise disjoint open subintervals of $(a, b)$ that is finer than $\{J(\varepsilon(y), y)\}_{y \in C}$ (see (ii)) and such that $[a, b] \backslash \bigcup_{n} K_{n}$ is countable. Moreover, there exists a map $\Psi: \mathbb{N} \rightarrow C$ such that $K_{n} \subset J(\varepsilon(\Psi(n)), \Psi(n))$ for each $n \in \mathbb{N}$.

(iv) There is a map $\psi: \bigcup_{n} K_{n} \rightarrow[a, b]^{m}$ such that if $K_{n} \subset J\left(\varepsilon\left(y^{\prime}\right), y^{\prime}\right)$ where $\Psi(n)=y^{\prime}$ then for each $y \in K_{n}$ we have $\psi(y)=t \in T(y)$ and

$$
\forall i \in N_{m}: \quad t_{i} \in\left(\phi\left(y^{\prime}\right)_{i}-\delta, \phi\left(y^{\prime}\right)_{i}+\delta\right) .
$$

Proof. (i) The reader can easily verify that a point $x \in[a, b]$ is not (left, right) regular if and only if $f$ is constant on some neighbourhood of $x$.

Let us prove (ii) when $y^{\prime} \in C_{\text {lreg }}(f)$ (the other cases are similar). Since $y^{\prime} \in C_{\text {lreg }}(f)$, for $\phi\left(y^{\prime}\right) \in T\left(y^{\prime}\right)$ and $\delta$ defined above the set $f\left(\left(\phi\left(y^{\prime}\right)_{i}-\delta\right.\right.$, $\left.\phi\left(y^{\prime}\right)_{i}+\delta\right)$ ) is a left neighbourhood of $y^{\prime}$ for each $i \in N_{m}$. Now we can choose $\varepsilon\left(y^{\prime}\right)$ sufficiently small to satisfy

$$
J\left(\varepsilon\left(y^{\prime}\right), y^{\prime}\right) \subset \bigcap_{i=0}^{m-1} f\left(\left(\phi\left(y^{\prime}\right)_{i}-\delta, \phi\left(y^{\prime}\right)_{i}+\delta\right)\right),
$$

which proves (ii) for $y^{\prime} \in C_{\text {lreg }}(f)$.

Let us show (iii). Notice that if $(a, b) \backslash C$ is countable then so is $A=$ $(a, b) \backslash \bigcup_{y \in C} J(\varepsilon(y), y)$. Indeed, $A \subset(A \cap C) \cup([a, b] \backslash C)$ and $A \cap C$ is countable by Lemma 2.2(i). Now (iii) is a consequence of Lemma 2.2(ii). The existence of $\Psi$ comes from the fact that $\left\{K_{n}\right\}_{n}$ is finer than $\{J(\varepsilon(y), y)\}_{y \in C}$.

Property (iv) is an easy consequence of (ii) and (iii).

3. Properties of maps from $L^{\sharp}(m,[a, b]), m \in\{\infty, 2,3, \ldots\}$. In what follows for $f \in C([a, b])$ we use the notation

$$
\begin{aligned}
& B_{1}(f)=\{x \in[a, b]: f(y) \geq f(x), \forall y \in[a, x] \& f(x) \geq f(y), \forall y \in[x, b]\}, \\
& B_{2}(f)=\{x \in[a, b]: f(y) \leq f(x), \forall y \in[a, x] \& f(x) \leq f(y), \forall y \in[x, b]\},
\end{aligned}
$$

and $B(f)=B_{1}(f) \cup B_{2}(f)$. If there is no ambiguity we often write $B_{i}$, resp. $B$ instead of $B_{i}(f)$, resp. $B(f)$. 


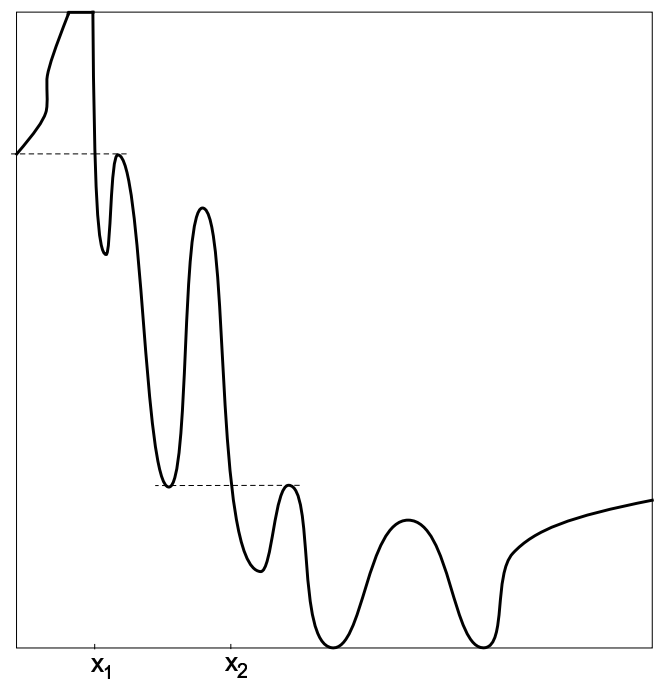

Fig 3. $f \in L(2,[a, b])$ and $x_{1}, x_{2} \in B_{1}(f)$

For $f \in L(m,[a, b])$ and $y \in[a, b]$ we put $m_{y}=m_{y}(f)=\min f^{-1}(y)$ and $M_{y}=M_{y}(f)=\max f^{-1}(y)$. The closed sets $S_{0}=S_{0}(f), S_{m-1}=S_{m-1}(f)$, $S_{(0, m-1)}=S_{(0, m-1)}(f)$ are defined as

$$
S_{0}=\overline{\left\{m_{y}: y \in[a, b]\right\}},
$$

$$
S_{m-1}=\overline{\left\{M_{y}: y \in[a, b]\right\}}, \quad S_{(0, m-1)}=S_{0} \cap S_{m-1} .
$$

Since for every $m \in\{\infty, 2,3,4, \ldots\}$

$$
L(m,[a, b]) \subset L^{\sharp}(m,[a, b]) \subset L(2,[a, b])
$$

we can apply the results developed in [B1] for maps from $L(2,[a, b])$.

Lemma 3.1 ([B1]). Let $f \in L(2,[a, b])$ and $S_{(0, m-1)} \neq \emptyset$.

(i) Either $B_{1}$ or $B_{2}$ is empty, hence $B \in\left\{B_{1}, B_{2}\right\}$.

(ii) $S_{(0, m-1)} \subset B \backslash\{a, b\}$.

(iii) The closed set $B$ can be expressed as a union $(n \geq 1)$

$$
\left\{b_{n}\right\}_{n<\mathcal{K}} \cup \bigcup_{n<\mathcal{L}}\left[b_{n}^{-}, b_{n}^{+}\right],
$$

where $b_{n}^{-}<b_{n}^{+}$for each cardinal $n, 1 \leq n<\mathcal{L}$; in the topology of $[a, b]$, the points $a, b$ are not limit points of the set $\left\{b_{n}\right\}_{n<\mathcal{K}} \cup$ $\bigcup_{n<\mathcal{L}}\left\{b_{n}^{-}, b_{n}^{+}\right\}$and no point $b_{m} \in\left\{b_{n}\right\}_{n<\mathcal{K}}$ is a two-sided limit point of that set, hence $\mathcal{K}, \mathcal{L}$ are at most countable cardinals.

(iv) If $\omega_{f} \subset B_{1}$ is an $\omega$-limit set then either $\omega_{f}=\{p\}$ and $p \in \operatorname{Fix}(f)$, or $\omega_{f}$ is a periodic orbit of period 2.

(v) If $\omega_{f} \subset B_{2}$ is an $\omega$-limit set then $\omega_{f}=\{p\}$ and $p \in \operatorname{Fix}(f)$. 
(vi) $a \in B_{1}\left(a \in B_{2}\right)$, resp. $b \in B_{1}\left(b \in B_{2}\right)$ if and only if $f(a)=b$ $(f(a)=a)$, resp. $f(b)=a(f(b)=b)$.

(vii) $\operatorname{card}\left(B_{1} \cap \operatorname{Fix}(f)\right) \leq 1$.

Lemma 3.2. Let $f \in L(2,[a, b])$.

(i) If $B_{1}(f)=B_{2}(f)=\emptyset$ then $S_{(0, m-1)}=\emptyset$.

(ii) If $B_{1}(f) \neq \emptyset \neq B_{2}(f)$ then for some $a \leq a_{1}<b_{1} \leq b$ either $f\left(\left[a, a_{1}\right]\right)=\{a\}, f\left(\left[b_{1}, b\right]\right)=\{a\}, B_{2}(f)=\left[a, a_{1}\right], B_{1}(f)=\left[b_{1}, b\right]$, or $f\left(\left[a, a_{1}\right]\right)=\{b\}, f\left(\left[b_{1}, b\right]\right)=\{b\}, B_{1}(f)=\left[a, a_{1}\right], B_{2}(f)=\left[b_{1}, b\right]$. In any case $S_{(0, m-1)}=\emptyset$.

Proof. Property (i) is a consequence of Lemma 3.1(ii). For (ii) see [B, Cor. L.2.1].

We have seen that for $f \in L^{\sharp}(m,[a, b])$ if $\omega_{f}(x) \subset B$ then $\omega_{f}(x)$ has a simple structure. In fact it is a periodic orbit and $\operatorname{card} \omega_{f}(x) \leq 2$. However, the number of different $\omega$-limit sets that are subsets of $B$ can be infinite. Fortunately, for each such $f$ one can consider a simplified version $g$ of $f$ (more precisely, a factor $([a, b], g)$ of $([a, b], f))$ which is in $L^{\sharp}(m,[a, b])$ again and has a very poor structure of $\omega$-limit sets in $B(g)$. The precise statement is given in Lemma 3.3.

Now we introduce some useful notation. For intervals $J=[\alpha, \beta] \subset[a, b]$ and $K=[\gamma, \delta] \subset[a, b]$, where $a \leq \alpha<\gamma \leq \delta<\beta \leq b$, the symbol $h(J, K)$ denotes a continuous nondecreasing piecewise affine map from $[a, b]$ onto $[a, b]$ that is constant on $[a, \alpha], K$ and $[\beta, b]$.

Lemma 3.3. Let $f \in L^{\sharp}(m,[a, b])$ and assume that $\emptyset \neq B(f) \in\left\{B_{1}(f)\right.$, $\left.B_{2}(f)\right\}$. There is a map $g \in L^{\sharp}(m,[a, b])$ such that $([a, b], g)$ is a factor of $([a, b], f)$ and one of the following possibilities holds.

(i) $B_{2}(g)=\emptyset$ and if $\omega_{g}(x) \subset B_{1}(g)$ then either $\omega_{g}(x)=\{a, b\}$ or $\omega_{g}(x)=\{p\}$ for some $p \in \operatorname{Fix}(g) \cap B_{1}(g)$.

(ii) $B_{1}(g)=\emptyset$ and if $\omega_{g}(x) \subset B_{2}(g)$ then $\omega_{g}(x)=\{p\}$ for some $p \in$ $\operatorname{Fix}(g) \cap\{a, b\}$.

Proof. Without loss of generality we can assume that $B_{1}(f) \neq \emptyset$ and $B_{2}(f)=\emptyset$. We show that (i) holds in this case. Set

$$
D=\left\{(x, f(x)) \in B_{1} \times B_{1}: f^{2}(x)=x<f(x)\right\} \cup\{(a, b)\} .
$$

By Lemma 3.1(vii),(iv) there is nothing to prove if $D=\{(a, b)\}$. In this case we put $g=f$.

For $(u, v) \in D$ we can consider a uniquely determined factor $\left([a, b], f_{u}\right)$ of $([a, b], f)$ with a factor map $h(J, K)$, where $\alpha=u, \beta=v$ and $\gamma=\delta$. Now,

$$
y:=\max \left\{u:(u, v) \in D \& f_{u} \in L^{\sharp}(m,[a, b])\right\}
$$


exists, since otherwise $f \notin L^{\sharp}(m,[a, b])$. Define

$$
D_{1}=\{x: x>y \&(x, f(x)) \in D\} .
$$

If $D_{1}=\emptyset$, we can put $g=f_{y}$. Otherwise $z:=\min D_{1}$ exists, $z>y$ and for $(y, \widetilde{y}),(z, f(z)) \in D$ there is a factor $([a, b], g)$ of $([a, b], f)$ with a factor map $h(J, K)$, where $\alpha=y<\gamma=z<\delta=f(z)<\beta=\widetilde{y}$.

Summarizing, at least one of the following possibilities holds: (i) $g=f$, (ii) $g(a)=b, g(b)=a$, (iii) $g(c)=c$, where $c=h(J, K)(\gamma) \in(a, b)$. This implies that $B_{2}(g)=\emptyset$.

Obviously, $g \in L^{\sharp}(m,[a, b])$ and from Lemma 3.1(iv) and our choice of $y$ and $z$ satisfying $D_{1} \cap(y, z)=\emptyset$ property (i) follows.

If $B_{2}(f) \neq \emptyset$ and $B_{1}(f)=\emptyset$ then the existence of $g$ satisfying (ii) can be shown similarly.

For $g \in L^{\sharp}(m[a, b])$ consider the following four properties (A)-(D):

$$
\text { (A) }\left\{\begin{array}{l}
\text { (A) } B_{1}(g)=B_{2}(g)=\emptyset ; \\
\text { (B) } B_{1}(g) \neq \emptyset \neq B_{2}(g) \\
\text { (C) } g \text { satisfies the conclusion of Lemma 3.3(i); } \\
\text { (D) } g \text { satisfies the conclusion of Lemma 3.3(ii). }
\end{array}\right.
$$

We set

$L^{\star}(m,[a, b])=\left\{g \in L^{\sharp}(m,[a, b]): g\right.$ has some of properties (A)-(D) $\}$.

Lemma 3.4. Let $g \in L^{\star}(m,[a, b])$. There is a positive integer $k_{0}=k_{0}(g)$ $\geq 2$ such that for any $x \in B(g)$ we have

$$
g^{k}(x) \in([a, b] \backslash B(g)) \cup(\operatorname{Fix}(g) \cap B(g)) \cup\{a, b\} \quad \text { for some } k<k_{0} .
$$

Proof. The statement is true for $g$ satisfying (A). For (B), use Lemma 3.2(ii). Now, suppose (C) holds. By Lemma 3.1(iii) the endpoints $a, b$ are not limit points of $\left\{b_{n}\right\}_{n<\mathcal{K}} \cup \bigcup_{n<\mathcal{L}}\left\{b_{n}^{-}, b_{n}^{+}\right\}$. By the same lemma, if $\operatorname{Fix}(g) \cap$ $B_{1}(g) \neq \emptyset$ then no point in this set is a two-sided limit point of $B_{1}(g)$. Since by our assumption $B_{1}(g)$ contains no other $\omega$-limit set (a 2-cycle), there is a $k_{0} \geq 2$ such that $B(g) \backslash((\operatorname{Fix}(g) \cap B(g)) \cup\{a, b\})$ contains at most $k_{0}$ consecutive iterates of any point of $B(g)$. The case when $g$ satisfies (D) can be verified similarly.

The next lemma uses the notation introduced in Section 2 before Lemma 2.3 .

Lemma 3.5. Let $g \in L^{\star}(m,[a, b])$. Then $(a, b) \backslash\left(C_{\text {lreg }}(f) \cup C_{\text {rreg }}(f) \cup\right.$ $\left.C_{\text {reg }}(f)\right)$ is countable.

Proof. Lemma 2.1 implies that it is sufficient to show

$$
(a, b) \backslash\left(C_{\text {lreg }}(f) \cup C_{\text {rreg }}(f) \cup C_{\text {reg }}(f)\right) \subset C_{\text {inter }}(f) \cup C_{\text {extrem }}(f) \cup L,
$$


where $L$ is the countable set given in $\left(1_{m}^{\sharp}\right)$ of the introduction. Take $y \in$ $(a, b) \backslash\left(C_{\text {lreg }}(f) \cup C_{\text {rreg }}(f) \cup C_{\text {reg }}(f)\right)$ and suppose that $y \notin C_{\text {inter }}(f) \cup L$. Then card $f^{-1}(y) \geq m$ and Lemma 2.3(i) shows that card $f^{-1}(y)$ is finite. Since $y \notin C_{\text {reg }}(f)$ there exists an $x \in f^{-1}(y)$ which is not regular. Since $x$ is an isolated point of $f^{-1}(y)$ we have $f(x)=y \in C_{\text {extrem }}(f)$.

Let $g \in L^{\star}(m,[a, b])$. We define closed sets $S_{0}=S_{0}(g), S_{1}=S_{1}(g), \ldots$, $S_{m-1}=S_{m-1}(g)$ as follows: $S_{0}, S_{m-1}$ are as in (4). By Lemma 3.5 we can use the pairwise disjoint countable system $\left\{K_{n}\right\}_{n}$ and the map $\psi: \bigcup_{n} K_{n} \rightarrow$ $[a, b]^{m}$ from Lemma 2.3(iii),(iv). For each $i \in\{1, \ldots, m-2\}$ we put

$$
S_{i}=S_{i}(g)=\overline{\left\{\psi(y)_{i}: y \in \bigcup_{n} K_{n}\right\}} .
$$

Also we put

$$
S=S(g)=\bigcap_{i=0}^{\infty} g^{-i}\left(S_{0} \cup S_{1} \cup \cdots \cup S_{m-1}\right) .
$$

The reader can verify that since $g \in L^{\star}(m,[a, b])$, by Lemmas 3.5 and 2.3 the sets $S_{i}, i \in N_{m}$, satisfy $[a, b]=\bigcap_{i=0}^{m-1} g\left(S_{i}\right) \supset \bigcup_{i=0}^{m-1} S_{i}$. There are a finite number of nontrivial intersections of elements of $\mathcal{H}=\left\{S_{0}, S_{1}, \ldots, S_{m-1}\right\}$, i.e., of sets

$$
S_{(i(1), \ldots, i(q))}=\bigcap_{j=1}^{q} S_{i(j)}, \quad 0 \leq i(1)<\cdots<i(q) \leq k-1 \& 2 \leq q \leq k .
$$

We define the kernel of $\mathcal{H}$ by Ker $\mathcal{H}=\bigcup_{i(1) \neq i(2)} S_{(i(1), i(2))}$, and the center of $\mathcal{H}$ by Cen $\mathcal{H}=\bigcap_{i=0}^{m-1} S_{i}$. Clearly, both Ker $\mathcal{H}$ and Cen $\mathcal{H}$ are closed.

Lemma 3.6. Let $g \in L^{\star}(m,[a, b]), \mathcal{H}=\left\{S_{0}, S_{1}, \ldots, S_{m-1}\right\}$, and $\operatorname{Ker} \mathcal{H}$ be as above. Then $g(\operatorname{Ker} \mathcal{H})$ is countable.

Proof. By our construction of $S_{0}, \ldots, S_{m-1}$, if $x \in S_{(i, j)}$ for $i \neq j$ then $g(x) \in[a, b] \backslash \bigcup_{n} K_{n}$, which is a countable set by Lemma 2.3(iii).

4. The proof of the main result. As before, for $g \in L^{\star}(m,[a, b])$ we consider the closed sets $S_{0}(g), \ldots, S_{m-1}(g)$ and also the set $S=S(g)$ given by (5). If $x \in S$ then by its itinerary with respect to $S_{0}, S_{1}, \ldots, S_{m-1}$ we mean any $\omega \in \Omega_{m}$ such that $g^{i}(x) \in S_{\omega_{i}}$ for $i \in \mathbb{N} \cup\{0\}$. For $M \subset S$ we denote by $\Omega(M)$ the least closed $\sigma$-invariant subset of $\Omega_{m}$ that contains all possible itineraries of points of $M$ with respect to $S_{0}, S_{1}, \ldots, S_{m-1}$. In particular, if $M=\operatorname{Fix}(f) \cap \operatorname{Cen} \mathcal{H} \neq \emptyset$ then $\Omega(M)=\Omega_{m}$, hence $\operatorname{ent}(\sigma, \Omega(M))=\log m$.

For $g \in L^{\star}(m,[a, b])$ we fix the value $k_{0}=k_{0}(g) \geq 2$ given by Lemma 3.4. Here is the key lemma: 
Lemma 4.1. Let $g \in L^{\star}(m,[a, b])$. If $M \subset S$ is minimal and $M \neq$ $\operatorname{Fix}(g) \cap \operatorname{Cen} \mathcal{H}$ then

$$
\operatorname{ent}(\sigma, \Omega(M)) \leq \max \left(\operatorname{ent}(g, M), \frac{1}{k_{0}} \log (m-1)+\frac{k_{0}-1}{k_{0}} \log m\right) .
$$

Proof. Put $X=\left\{(x, \omega): x \in M \& g^{i}(x) \in S_{\omega_{i}}\right.$ for each $\left.i \in \mathbb{N} \cup\{0\}\right\}$. The map $G=g \times \sigma$ defined by $G(x, \omega)=(g(x), \sigma(\omega))$ is continuous on the compact metric space $X$ (with respect to the product metric). Moreover, the dynamical system $(M, g)$, resp. $(\Omega(M), \sigma)$ is a factor of $(X, G)$ given by the (factor map) projection $\Pi_{1}: X \rightarrow M$, resp. $\Pi_{2}: X \rightarrow \Omega(M)$. Using Theorem 1.1 we see that

$$
\operatorname{ent}(\sigma, \Omega(M)) \leq \operatorname{ent}(G) \leq \operatorname{ent}(g, M)+\sup _{x \in M} \operatorname{ent}\left(G, \Pi_{1}^{-1}(\{x\})\right) .
$$

Moreover, $\Lambda_{x}=\Pi_{2}\left(\Pi_{1}^{-1}(\{x\})\right)$ is a closed subset of $\Omega_{m}$ whenever $x \in M$. By (2) we have

$$
\operatorname{ent}\left(G, \Pi_{1}^{-1}(\{x\})\right)=\lim _{n} \frac{1}{n} \log \operatorname{card} \Lambda_{x}(n) .
$$

Concerning the relationship of the sets $M$, Cen $\mathcal{H}$, Ker $\mathcal{H}$ we consider several possibilities (see Lemma 1.2).

Case I: $M$ is a cycle. Then $\operatorname{ent}(g, M)=0$ and to prove (6) we need to verify that

$$
\operatorname{ent}(\sigma, \Omega(M)) \leq \frac{1}{k_{0}} \log (m-1)+\frac{k_{0}-1}{k_{0}} \log m .
$$

CASE I(a): $M \cap$ Cen $\mathcal{H}=\emptyset$. This is true if $g$ satisfies (A) or (B) of ( $\mathbf{\$}$ ) (see Lemma 3.2). Our assumption implies that for each $x \in M$ and positive integer $n$ we have card $\Lambda_{x}(n) \leq(m-1)^{n}$, hence (8) yields ent $\left(G, \Pi_{1}^{-1}(\{x\})\right) \leq$ $\log (m-1)$. Now the property (6) is a consequence of $(7)$ and of the inequality $\log (m-1) \leq \frac{1}{k_{0}} \log (m-1)+\frac{k_{0}-1}{k_{0}} \log m$.

CASE I(b): $M \cap$ Cen $\mathcal{H} \neq \emptyset$. Then $g$ satisfies (C) or (D) of (\$). Moreover, $\emptyset \neq \operatorname{Cen} \mathcal{H} \subset S_{(0, m-1)} \subset B \backslash\{a, b\}$ by Lemma 3.1(ii). Since $M \neq \operatorname{Fix}(g) \cap$ Cen $\mathcal{H}$, using Lemma 3.3 we obtain $M \backslash B \neq \emptyset$. By Lemma 3.4, for each $n \in \mathbb{N}$,

$$
\operatorname{card} \Omega(M)(n) \leq \operatorname{card} M \cdot(m-1)^{n / k_{0}} m^{n-n / k_{0}},
$$

hence $\operatorname{ent}(\sigma, \Omega(M)) \leq \frac{1}{k_{0}} \log (m-1)+\frac{k_{0}-1}{k_{0}} \log m$ by (2). Thus, (6) is true in this case.

CASE II: $M$ is infinite. In this case we show that ent $\left(G, \Pi_{1}^{-1}(\{x\})\right)=0$ for each $x \in M$. Then from (7) we will obtain $\operatorname{ent}(\sigma, \Omega(M)) \leq \operatorname{ent}(g, M)$, proving (6). 
Fix $x \in M$, put $C=M \cap \operatorname{Ker} \mathcal{H}$ and $\widetilde{C}=M \cap g(\operatorname{Ker} \mathcal{H})$, and set, as in the proof of Lemma 1.2(iv), $C(n, x)=\operatorname{card}\left\{0 \leq i \leq n-1: g^{i}(x) \in C\right\}$ and $\widetilde{C}(n, x)=\operatorname{card}\left\{0 \leq i \leq n-1: g^{i}(x) \in \widetilde{C}\right\}$. Clearly $C(n, x) \leq \widetilde{C}(n+1, x)$ for each $n$. If $s(n, \varepsilon)=s\left(n, \varepsilon, \Pi_{1}^{-1}(\{x\})\right)$ denotes the maximal cardinality of an $(n, \varepsilon)$-separated subset of $\Pi_{1}^{-1}(\{x\})$ (with respect to $G$ ), by the definition of Ker $\mathcal{H}$ we have $s(n, \varepsilon) \leq m^{C(n, x)}$ for any sufficiently small $\varepsilon$. It follows from Lemmas 3.6 and 1.2(iv) that

$$
\limsup _{n \rightarrow \infty} \frac{1}{n} \log s(n, \varepsilon) \leq \lim _{n \rightarrow \infty} \frac{1}{n} \log m^{C(n, x)} \leq \lim _{n \rightarrow \infty} \frac{1}{n} \log m^{\widetilde{C}(n+1, x)}=0,
$$

hence $\operatorname{ent}\left(G, \Pi_{1}^{-1}(\{x\})\right)=0$.

Corollary 4.2. Under the assumptions of Lemma 4.1,

$$
\operatorname{ent}(\sigma, \Omega(M)) \leq \max \left(\operatorname{ent}(g), \frac{1}{k_{0}} \log (m-1)+\frac{k_{0}-1}{k_{0}} \log m\right) .
$$

Proof. By the definition, ent $(g, M) \leq \operatorname{ent}(g)$. Now apply Lemma 4.1.

As before, we use the notation $N_{m}=\{0,1, \ldots, m-1\}$.

Definition. Let $\Omega \subset \Omega_{m}$ and $j, k \in \mathbb{N}, j \leq k$. We say that $\omega(k) \in \Omega(k)$ contains $\omega=\left(\omega_{0}, \ldots, \omega_{j-1}\right) \in N_{m}^{j}$ if for some $l \in\{0, \ldots, k-j\}$ and each $i \in\{0, \ldots, j-1\}$,

$$
\omega(k)_{l+i}=\omega_{i} .
$$

Definition. Let $g \in L^{\star}(m,[a, b])$. We will say that $\omega=\left(\omega_{0}, \ldots, \omega_{j-1}\right)$ $\in N_{m}^{j}$ is a $j$-itinerary of $x \in[a, b]$ if $g^{i}(x) \in S_{\omega_{i}}(g)$ for $i \in\{0, \ldots, j-1\}$. We say that a $j$-itinerary of $x$ does not exist if $\left\{x, \ldots, g^{j-1}(x)\right\} \nsubseteq S_{0}(g) \cup$ $S_{1}(g) \cup \cdots \cup S_{m-1}(g)$.

Combining Lemma 4.1 and Corollary 4.2 with the results of Sections 1 and 2 we now obtain the main result of this paper.

TheOREM 4.3. Let $f \in L^{\sharp}(m,[a, b])$. Then the topological entropy of $f$ is greater than or equal to $\log m$. In particular, this is true for any map from $L(m,[a, b]) \subset L^{\sharp}(m,[a, b])$.

Proof. Let $f \in L^{\sharp}(m,[a, b])$. There is nothing to prove if $\operatorname{Ker} \mathcal{H}=\emptyset$. In this case $\left.S_{0}(f), S_{1}(f), \ldots, S_{m-1}(f)\right\}$ form an $m$-horseshoe and so ent $(f) \geq$ $\log m$ by Proposition 0.2 .

Now, suppose Ker $\mathcal{H} \neq \emptyset$. By Lemmas 3.3 and 3.4, instead of $f$ we can consider the map $g \in L^{\star}(m,[a, b])$ such that $\operatorname{ent}(f) \geq \operatorname{ent}(g)$. Obviously it is sufficient to prove ent $(g) \geq \log m$.

In what follows all sets are taken with respect to $g$. The inequality $\operatorname{ent}(g) \geq \log m$ is clear if Ker $\mathcal{H}=\emptyset$ since in this case the sets $S_{0}, S_{1}, \ldots, S_{m-1}$ form an $m$-horseshoe. 
Suppose to the contrary that $\operatorname{Ker} \mathcal{H} \neq \emptyset$ and $\operatorname{ent}(g)<\log m$. Let $k_{0} \geq 2$ be as in Lemma 3.4. Using Theorem 1.3 we can consider a minimal set $\Gamma$ in $\Omega_{m}$ such that

$$
\operatorname{ent}(\sigma, \Gamma)>\max \left(\operatorname{ent}(g), \frac{1}{k_{0}} \log (m-1)+\frac{k_{0}-1}{k_{0}} \log m\right) .
$$

Lemma 1.2(i) shows that for each $x \in \operatorname{Ker} \mathcal{H}$ there is a minimal set $M(x)$ in $[a, b]$ such that $M(x) \subset \omega_{g}(x)$.

Put $B_{S}=\{x \in S \cap \operatorname{Ker} \mathcal{H}: M(x) \neq \operatorname{Fix}(g) \cap \operatorname{Cen} \mathcal{H}\}$ (see (5) for $S$ ). We deduce from Lemma 4.1 that (6) is true for $M(x)$ and ent $(\sigma, \Omega(M(x)))$ when $x \in B_{S}$. Hence by the minimality of $\Gamma$, Lemma 1.4 and (9) (for $x=\infty$ see $(3))$,

$$
\forall x \in B_{S} \cup\{\infty\}: \quad \Omega(M(x)) \cap \Gamma=\emptyset .
$$

Since $\Gamma$ is $\sigma$-invariant we even see that for each $x \in B_{S} \cup\{\infty\}$ there is $n(x) \in \mathbb{N}$ such that

$$
\text { no } \gamma \in \Gamma(m) \text { contains } \omega(n(x))
$$

whenever $m \geq n(x)$ and $\omega(n(x)) \in \Omega(M(x)(n(x))$.

Now we define an open cover $\{U(x)\}_{x \in \operatorname{Ker} \mathcal{H}}$ of Ker $\mathcal{H}$ in three steps:

(i) If $x \in(\operatorname{Ker} \mathcal{H}) \backslash S$ and $g^{m(x)}(x) \notin S_{0} \cup S_{1} \cup \cdots \cup S_{m-1}$, choose $U(x)$ in such a way that $g^{m(x)}(U(x)) \cap\left(S_{0} \cup S_{1} \cup \cdots \cup S_{m-1}\right)=\emptyset$.

(ii) If $x \in B_{S}$ then we can consider $m(x) \in \mathbb{N}$ such that for any itinerary $\omega$ of $x, \omega(m(x))$ contains some element of $\Omega(M(x))(n(x))$; now, using the continuity of $g$, choose a neighbourhood $U(x)$ of $x$ such that for any $y \in U(x)$ either the $m(x)$-itinerary of $y$ does not exist or for any itinerary $\omega$ of $y$, $\omega(m(x))$ contains some element of $\Omega(M(x))(n(x))$.

(iii) Let $x \in S \cap \operatorname{Ker} \mathcal{H}$ be such that $M(x)=\operatorname{Fix}(g) \cap \operatorname{Cen} \mathcal{H}=\{p\}$. Since Cen $\mathcal{H} \subset S_{(0, m-1)}$, from Lemmas 3.1(ii) and 3.3(i) we get $p \in B_{1} \cap(a, b)$. We know that card $g^{-1}(p) \geq 2$. Let $z \in g^{-1}(p) \backslash\{p\}$. Using the definition (4) of $S_{0}, S_{m-1}$ the reader can verify that if $z<p$, resp. $z>p$ then for some small positive $\eta$ we have $S_{0} \cap(p-\eta, p)=\emptyset$, resp. $S_{m-1} \cap(p, p+\eta)=\emptyset$. Therefore we can consider $m(x) \in \mathbb{N}$ and $U(x)$ such that for any $y \in U(x)$ either $g^{i}(y)=p$ for some $i \leq m(x)$, or the $m(x)$-itinerary of $y$ does not exist, or for any itinerary $\omega$ of $y, \omega(m(x))$ contains some element of $\Omega(M(\infty))(n(\infty))$.

Obviously we have found the pairs $U(x), m(x)$, where $\{U(x)\}_{x \in \operatorname{Ker} \mathcal{H}}$ is an open cover of the compact set $\operatorname{Ker} \mathcal{H}$; let $\left\{U\left(x_{1}\right), \ldots, U\left(x_{k}\right)\right\}$ be its finite subcover, and put

$$
k^{\star}=\max \left\{m\left(x_{1}\right), \ldots, m\left(x_{k}\right)\right\} .
$$

To finish the proof we define

$$
R_{i}=S_{i} \backslash(\operatorname{Fix}(g) \cap \operatorname{Cen} \mathcal{H}), \quad i \in N_{m} .
$$


Since $\bigcap_{i=0}^{m-1} g\left(R_{i}\right) \supset \bigcup_{i=0}^{m-1} R_{i}$, for each $l \in \mathbb{N}$ and $\gamma \in \Gamma(l)$ there is $x=$ $x(\gamma) \in \bigcup_{i=0}^{m-1} R_{i}$ such that for each $i \in N_{l}$ we have

$$
g^{i}(x) \in R_{\gamma_{i}}, \quad g^{i}(x) \notin \operatorname{Fix}(g) \cap \operatorname{Cen} \mathcal{H} .
$$

It is clear that the sets $T_{i}=R_{i} \backslash \bigcup_{j=1}^{k} U\left(x_{j}\right), i \in N_{m}$, are closed. Moreover,

$$
\delta=\min \left\{\operatorname{dist}\left(T_{i}, T_{j}\right): i \neq j\right\}>0 .
$$

Suppose that for some $l>k^{\star}, \gamma \in \Gamma(l), x(\gamma)$ and $i \in\left\{0, \ldots, l-1-k^{\star}\right\}$ we have $g^{i}(x(\gamma)) \in U\left(x_{j}\right)$. Then by definition of $\{U(x)\}_{x \in \operatorname{Ker} \mathcal{H}}$ either the $k^{\star}$-itinerary of $g^{i}(x(\gamma))$ does not exist, or $\gamma$ contains some element of $\Omega\left(M\left(x_{j}\right)\right)\left(n\left(x_{j}\right)\right)$, which is impossible by (11) and (10). This implies that for any $l>k^{\star}, \gamma \in \Gamma(l)$ and $x(\gamma)$ we have

$$
\left\{g^{i}(x(\gamma))\right\}_{i=0}^{l-1-k^{\star}} \subset T_{0} \cup T_{1} \cup \cdots \cup T_{m-1} .
$$

Now, estimating the topological entropy of $g$ we have, for some $\varepsilon<\delta$ and each $l>k^{\star}$,

$$
s\left(l-1-k^{\star}, \varepsilon,[a, b]\right) \geq \operatorname{card} \Gamma(l) / m^{k^{\star}},
$$

hence by $(9)$ and $(2)$, ent $(g) \geq \operatorname{ent}(\sigma, \Gamma)>\operatorname{ent}(g)$ - a contradiction. The proof of our theorem is finished.

5. The topological entropy of a Besicovitch function. For the Lebesgue measure $\lambda$ we define

$$
C(\lambda)=\left\{f \in C([0,1]): \forall \text { Borel } A \subset[0,1]: \lambda(A)=\lambda\left(f^{-1}(A)\right)\right\} .
$$

By a Besicovitch function we mean a function which has a unilateral derivative (finite or infinite) at no point. In [B2], [B3] we have constructed Besicovitch functions in $C(\lambda)$. Now we show that such maps have an infinite topological entropy. First, let us repeat the construction from [B2]. Also we correct an inaccuracy there (compare the definition of $\phi$ ).

Construction. Let $k>4$. Set

$$
D=[0,1 / 2] \backslash L, \quad \text { where } \quad L=\bigcup_{m=1}^{\infty} \bigcup_{p=1}^{2^{m-1}} r_{m, p},
$$

and the open intervals $r_{m, p}=\left(a_{m, p}, b_{m, p}\right)$ are constructed as follows:

( $\alpha) d_{1,1}=[0,1 / 2], r_{1,1} \subset d_{1,1}, \lambda\left(r_{1,1}\right)=1 / 2 k, b_{1,1}$ is the centre of $d_{1,1}$;

$(\beta)$ if $d_{n, 1}, \ldots, d_{n, 2^{n-1}}$ are the intervals of $[0,1 / 2] \backslash \bigcup_{q=1}^{n-1} \bigcup_{p=1}^{2^{q-1}} r_{q, p}$ for $n>1$ (from left to right), then $r_{n, p} \subset d_{n, p}, b_{n, p}$ is the centre of $d_{n, p}$ and $\lambda\left(r_{n, p}\right)=1 / 2 k^{n}$.

Obviously, $\lambda(L)=1 / 2(k-2)$ and $\lambda(D)=(k-3) / 2(k-2)$. 
Let $\phi:[0,1 / 2] \rightarrow[0,1]$ be a nondecreasing continuous function such that $\phi(0)=0, \phi(1 / 2)=1, \phi$ is constant on every interval $r_{m, p}$, and $\phi\left(r_{m, p}\right)=$ $\left\{(2 p-1) / 2^{m}\right\}$. Define a function $p:[0,1] \rightarrow[0,1]$ by

$$
p(x)= \begin{cases}\phi(x), & x \in[0,1 / 2] \\ \phi(1-x), & x \in[1 / 2,1]\end{cases}
$$

The function $p$ and the interval $[0,1]$ form the well-known step triangle $[\mathrm{P}]$.

The above procedure will be called the construction of a step triangle with base $[0,1]$, height 1 and parameter $k$.

We have seen that the base $[0,1]$ lies below the vertex $(1 / 2,1)$-in such a case we say that the step triangle is positively oriented. The set $\{(x, p(x)): x \in[0,1 / 2]\}$, resp. $\{(x, p(x)): x \in[1 / 2,1]\}$ is the left, resp. right side of triangle. Further, put $u_{y}=\{(x, y): x \in[0,1]\}$ and let $g(f)$ be the graph of the function $f$.

Now, we can construct a function $f$ as follows:

$\left(c_{0}\right)$ construct a positively oriented step triangle with base [0,1], height 1 and parameter $k$; the sides of the step triangle define a function $f_{0}$;

$\left(c_{n}\right)$ for $n>0$, construct step triangles (positively or negatively oriented) whose bases are intervals of the set $\bigcup_{p=1}^{2^{n-1}} u_{2 p-1 / 2^{n}} \cap g\left(f_{n-1}\right)$, height $1 / 2^{n}$ and parameter $k$; the constructed triangles are placed inside the bigger triangle, with bases on its sides; the union of sides of all triangles constructed so far defines a function $f_{n}$.

Finally, put $f=\lim _{n \rightarrow \infty} f_{n}$ (obviously $\varrho\left(f_{n-1}, f_{n}\right)=1 / 2^{n}$ ).

Theorem 5.1 ([B2], [B3]). $f \in C(\lambda)$ and $f$ is a Besicovitch function.

In order to illustrate how our Theorem 4.3 can be used we will prove that $\operatorname{ent}(f)=\infty$. Since $\operatorname{ent}\left(f^{n}\right)=n \operatorname{ent}(f)$ for each $n \in \mathbb{N}$, by Theorem 4.3 it is sufficient to show that

Theorem 5.2. $f^{2} \in L^{\sharp}(\infty,[0,1])$.

Proof. Since $f(0)=f(1)=0$ and $f(1 / 2)=1$ we have $f^{2} \in L(2,[0,1])$. Put $M=\left\{p / 2^{n}: n \in \mathbb{N} \cup\{0\}, p \in\left\{0,1, \ldots, 2^{n}\right\}\right\}$ and suppose that $y \in$ $[0,1] \backslash M$. We will show that card $f^{-1}(y)=\infty$. Otherwise there would be the smallest step triangle $T$ such that $u_{y}$ has a nonempty intersection with its sides. Without loss of generality we can assume that this step triangle $T$ has a positive orientation, it is of height $1 / 2^{n}$ and has its base in $u_{(2 p-1) / 2^{n}}$. Since $y \notin M$ there is a unique positive integer $m$ such that for $L=\sum_{i=1}^{m} 1 / 2^{n+i}$ we have

$$
y \in\left(\frac{2 p-1}{2^{n}}+L-\frac{1}{2^{n+m}}, \frac{2 p-1}{2^{n}}+L\right) .
$$

Then from our construction it follows that $u_{y}$ has a nonempty intersection with sides of a negatively oriented step triangle (placed inside $T$ and with 
base on a side of $T$ ) of height $1 / 2^{n+m}$ and with base in $u_{(2 p-1) / 2^{n}+L}$. This is a contradiction.

Now, from $\left(f^{2}\right)^{-1}=f^{-1}\left(f^{-1}\right)$ we obtain

$$
\operatorname{card}\left(f^{2}\right)^{-1}(y) \begin{cases}\geq 2, & y \in M, \\ =\infty, & y \notin M .\end{cases}
$$

Since $M$ is countable the conclusion $f^{2} \in L^{\sharp}(\infty,[0,1])$ follows.

\section{References}

[BC] L. S. Block and W. A. Coppel, Dynamics in One Dimension, Lecture Notes in Math. 1513, Springer, Berlin, 1992.

[B1] J. Bobok, The topological entropy versus level sets for interval maps, Studia Math. 153 (2002), 249-261.

[B2] -, On non-differentiable measure-preserving functions, Real Anal. Exchange 16 (1990/91),119-129.

[B3] - On a space of Besicovitch functions, ibid., to appear in 2005.

[Bo] R. Bowen, Entropy for group endomorphisms and homogeneous spaces, Trans. Amer. Math. Soc. 153 (1971), 401-414.

[Br] A. M. Bruckner, Differentiation of Real Functions, CRM Monogr. Ser. 5, Amer. Math. Soc., Providence, RI, 1994.

[DGS] M. Denker, Ch. Grillenberger and K. Sigmund, Ergodic Theory on Compact Spaces, Lecture Notes in Math. 527, Springer, 1976.

[G] Ch. Grillenberger, Constructions of strictly ergodic systems, Z. Wahrsch. Verw. Gebiete 25 (1973), 323-334.

[Ly] M. Yu. Lyubich, Entropy of analytic endomorphisms of the Riemannian sphere, Funct. Anal. Appl. 15 (1981), 300-302.

[Ma] P. Maličký, Topological entropy and cardinalities of level sets, preprint 2003, $10 \mathrm{pp}$.

[MP] M. Misiurewicz and F. Przytycki, Topological entropy and degree of smooth mappings, Bull. Acad. Polon. Sci. Sér. Sci. Math. Astronom. Phys. 25 (1977), 573-574.

$[\mathrm{P}] \quad$ E. D. Pepper, On continuous functions without a derivative, Fund. Math. 12 (1928), 244-253.

\section{KM FSv ČVUT}

Thákurova 7

16629 Praha 6, Czech Republic

E-mail: erastus@mbox.cesnet.cz

Received January 13, 2003

Revised version June 29, 2004 